\title{
Long non-coding RNA BCYRN1 promotes the proliferation and metastasis of cervical cancer via targeting microRNA-138 in vitro and in vivo
}

\author{
JIE PENG, FANG HOU, JUN FENG, SHUI-XIAN XU and XIAO-YAN MENG \\ Department of Obstetrics and Gynecology, Wuzhong People's Hospital, Suzhou, Jiangsu 215128, P.R. China
}

Received March 31, 2017; Accepted November 6, 2017

DOI: $10.3892 / \mathrm{ol} .2018 .8015$

\begin{abstract}
Cervical cancer is one of the most malignant types of tumor and the fourth leading cause of cancer-associated mortality in females worldwide. High expression of brain cytoplasmic RNA 1 (BCYRN1) has been detected in various tumors. The present study aimed to investigate the effect of BCYRN1 in the viability and motility of cervical cancer, and the relevant mechanism. The results demonstrated that BCYRN1 was upregulated in cervical cancer tissues compared with normal tissues. Elevated levels of BCYRN1 were also detected in three human cervical cancer cell lines ( $\mathrm{SiHa}, \mathrm{HeLa}$ and CaSki) compared with non-cancerous ectocervical epithelial cell line (Ect1/E6E7). The expression of BCYRN1 was suppressed following transfection with small interfering RNA (siRNA) in HeLa cells. The silence of BCYRN1 significantly reduced cell viability and motility. Furthermore, microRNA (miR)-138 was predicted as a direct target of BCYRN1 and the expression of miR-138 was elevated in HeLa cells transfected with BCYRN1 siRNA. Subsequently, elevated levels of miR-138 were suppressed by transfection with miR-138 inhibitor in HeLa cells pretreated with BCYRN1 siRNA. The targeting association between BCYRN1 and miR-138 was supported by luciferase reporter assays. Additionally, BCYRN1 siRNA partially counteracted the effect of miR-138 inhibitor on promoting cell viability and mobility in $\mathrm{HeLa}$ cells. Finally, the in vivo experiment verified that BCYRN1 siRNA was able to prevent tumor growth, and reduced the
\end{abstract}

Correspondence to: Dr Jie Peng, Department of Obstetrics and Gynecology, Wuzhong People's Hospital, 61 North Dongwu Road, Suzhou, Jiangsu 215128, P.R. China

E-mail: jiepengwzp@126.com

Abbreviations: BCYRN1, brain cytoplasmic RNA 1; miR-138, microRNA 138; lncRNAs, long non-coding RNAs; siRNA, small interfering RNA; PCNA, proliferation cell nuclear antigen; MMP, matrix metalloproteinase; VEGF, vascular endothelial cell growth factor; qRT-PCR, quantitative real-time polymerase chain reaction; 3'-UTR, 3'-untranslated region; CCK-8, Cell Counting Kit-8

Key words: brain cytoplasmic RNA 1, miRNA-138, cervical cancer, proliferation, invasion expression of migration marker proteins metalloproteinase 2 and vascular endothelial cell growth factor, with enhanced expression levels of miR-138. These results suggest that lncRNA BCYRN1 promotes the proliferation and invasion of cervical cancer via targeting miR-138.

\section{Introduction}

Cervical cancer is one of the most frequently occurring malignant tumors and the fourth leading cause of cancer-related mortality in females worldwide, causing about 300,000 deaths every year. More than $75 \%$ of these cases and deaths occur in developing countries (1). The disproportionately high burden of cervical cancer in developing countries and elsewhere medically underserved populations is principally because of lacking screening that can prevention and detection of early-stage cervical cancer (2). Therefore, it is very important to study a method to reliably predict disease outcome and reduce risk of treatment failures.

Protein-coding genes account for only about two percent of the human genome, whereas the overwhelming majority of transcripts are non-coding RNAs (ncRNAs), including lncRNAs. LncRNAs are defined as $>200 \mathrm{nt}$ in length (3). Their roles include regulating gene expression at the epigenetic, transcriptional, and post-transcriptional level in cellular homeostasis (4). As we know that IncRNA studies are still in their infancy and the functions remain unclear. Up to now, more and more literature has proved that lncRNA expressions are involved in cancer metastasis $(5,6)$. Brain cytoplasmic RNA 1 (BCYRN1), also called BC200, ordinarily cannot be detected in normal tissue, except for in the primate nervous system (7). Accordance to the literature, lncRNA BCYRN1is strongly expressed in some carcinomas of the cervic, oesophagus and lung, but not detected in normal tissues $(8,9)$.

MicroRNAs (miRNAs), a class of short ncRNA molecules ranging in size from 19 to 25 nucleotides (nt), is one of the well-characterized classes of ncRNAs. miRNAs have been recognized as a new class of genes involved in human cancer and have recently been shown to be biomarkers of diagnostic, prognostic and therapeutic $(10,11)$. Several studies have identified some miRNAs that were significantly altered and have the potential to restrain proliferation of cervical cancer $(12,13)$. Previous studies suggest that miRNA-138 restrains cervical cancer cells proliferation (14). Although a 
large number of ncRNA research has focused on the regulation of protein-coding genes mediated by them, it suggests that miRNAs and lncRNAs can interact with each other, especially in transcriptional regulation.

In the present study, we demonstrated that BCYRN1 was upregulated in cervical cancer tissues and cell lines. BCYRN1 was then knockdown by transfecting small interfering RNA (siRNA) in HeLa cells. BCYRN1 siRNA suppressed the viability and mobility of cervical cancer in vitro and in vivo and may through targeting miR-138. BCYRN1 may serve as a novel target for cervical cancer treatment.

\section{Materials and methods}

Patients and materials. The experiments were undertaken with the understanding and written consent of each subject. Informed consent was obtained from each patient recruited, and the study protocol was approved by the Human Ethics Committee of Wuzhong People's Hospital in Suzhou (Jiangsu, China). Cervical cancer tissues were collected from 25 cases of cervical cancer patients who underwent curative surgery from January 2005 to May 2014 at Wuzhong People's Hospital in Suzhou (Jiangsu, China) (Table I). And normal cervix healthy subjects were as normal control. On removal of the surgical specimen, researchers immediately transferred the tissues to the surgical lab. Each sample was snap-frozen in liquid nitrogen before RNA analysis.

Cell culture. Three human cervical cancer cell lines (SiHa, HeLa and CaSki) were cultured in this study. They were purchased from Cell Bank of the Chinese Academy of Science (Shanghai, China). A non-cancerous ectocervical epithelial cell line (Ect1/E6E7) was purchased from the Health Science. All cells were cultured in DMEM (Gibco-BRL, Grand Island, NY, USA), including 10\% fetal bovine serum (FBS; Hyclone; Invitrogen, Camarillo, CA, USA), as well as $100 \mathrm{U} / \mathrm{ml}$ penicillin and $100 \mu \mathrm{g} / \mathrm{ml}$ streptomycin (Invitrogen, Carlsbad, CA, USA). Cells were maintained in a humidified incubator $\left(37^{\circ} \mathrm{C}\right.$, $5 \% \mathrm{CO}_{2}$ ).

RT-PCR analysis. Total RNA from cervical cancer tissues and cells was extracted using Trizol reagent (Invitrogen). RNA was transcribed into cDNAs using the PrimerScript one-step RT-PCR kit (Takara, Dalian, China). Then cDNA template was amplified by RT-PCR (SYBR Premix Dimmer Eraser kit; TaKaRa). GADPH expression level was as a standardization for each gene expression. The primer sequences were as follows: BCYRN1 forward, 5'-CTGGGCAATATAGCGAGAC-3' and reverse, 5'-TGC TTTGAGGGAAGTTACG-3'; GAPDH forward, 5'-GTCAACGGATTTGGTCTGTATT-3' and reverse, 5'-AGTCTTCTGGGTGGCAGTGAT-3'. The condition of RT-PCR was $95^{\circ} \mathrm{C}$ for $2 \mathrm{~min}$, followed by 40 cycles of $15 \mathrm{sec}$ at $95^{\circ} \mathrm{C}$, and at last $1 \mathrm{~min}$ at $55^{\circ} \mathrm{C}$. The $2^{-\Delta \Delta \mathrm{Ct}}$ method was used to calculate the relative expression fold change of mRNAs.

Northern blot analysis. Total RNAs were extracted by the TRIzol (Gibco-BRL) method. Poly(A) ${ }^{+}$RNA was isolated using an Oligotex mRNA midi kit (Qiagen $\mathrm{GmbH}$, Hilden, Germany) following the manufacturer's instructions. Analysis of RNA expression was performed by Northern blotting as previously described (15). Total RNA was fractionated on a denaturing $12 \%$ polyacrylamide gel containing $8 \mathrm{M}$ urea, transferred to Nytran N membrane (Schleicher and Schuell, Germany, Dassell, Germany) by capillary method and fixed by ultraviolet cross-linking. Prehybridization of the filters was carried out in $50 \%$ formamide, $0.5 \%$ SDS, 5X SSPE, $5 \mathrm{X}$ Denhardt's solution and $20 \mu \mathrm{g} / \mathrm{ml}$ sheared, denatured salmon sperm DNA. Filters were washed at $70^{\circ} \mathrm{C}$ in $2 \mathrm{X} \mathrm{SSC}$ and $1 \%$ SDS for 10 rain, in $0.2 X$ SSC and $0.5 \%$ SDS for the next 10 rain, and then in 0.1 X SSC and $0.1 \%$ SDS for $20 \mathrm{~min}$. The filter for detecting the EP4 mRNA was treated with $1 \mathrm{pg} / \mathrm{ml}$ RNase A at $20^{\circ} \mathrm{C}$ for $10 \mathrm{~min}$, and washed again at $70^{\circ} \mathrm{C}$ in $0.1 \mathrm{X} \mathrm{SSC}$ and $0.1 \%$ SDS for 10 rains.

Cell transfection. hsa-miRNA-138 mimic/negative-control mimic and hsa-miRNA-138 inhibitor/negative-control inhibitor were purchased from Genechem (Shanghai, China). The BCYRN1 siRNAs were synthesized by Invitrogen. BCYRN1 siRNA (targeted region) sequences were as follows: siRNA_1, CGCCUGUAAUCCCAGCUCUCA; siRNA_2, AUAAGCG UAACUUCCCUCAAA; siRNA_3 CGUAACUUCCCUCAA AGCAACAACC.pcDNA-3.1(+)-BCYRN1(lncRNA-BCYRN1), the overexpression plasmid of BCYRN1 was constructed from Genechem. According to the manufacturer's instructions, transfections were performed using the Lipofectamine 2000 kit (Invitrogen).

CCK-8 analysis. Cervical cancer cells were seeded in a 96-well plate $\left(5 \times 10^{3}\right.$ cells/well) for $24 \mathrm{~h}$. Then the cultured cells were transfected with miR-138 mimic or BCYRN1 siRNA for $48 \mathrm{~h}$. Then CCK- 8 reagent $(5 \mathrm{mg} / \mathrm{ml})$ was added to each well and incubated in dark at $37^{\circ} \mathrm{C}$ for $2 \mathrm{~h}$. Finally, the absorbance was determined with the wavelength of $490 \mathrm{~nm}$.

Flow cytometric analysis. Cells transfected with desired plasmid or negative control were plated in 6-well plates. After incubation for $48 \mathrm{~h}, 3 \mu \mathrm{g} / \mathrm{ml}$ Annexin V-FITC and $5 \mu \mathrm{g} / \mathrm{ml}$ propidium iodide was used to staining at RT for $30 \mathrm{~min}$ in the dark. Then cultures were collected and analyzed using a FACSCalibur $^{\mathrm{TM}}$ flow cytometer (BD Biosciences, San Jose, CA, USA) with Beckman CXP software (Beckman Coulter, Inc., Brea, CA, USA).

Western blot analysis. The proteins, extracted from tissues and cultured cells, were separated through SDS-PAGE and then were transferred onto polyvinylidene fluoride (PVDF) membranes (Millipore, Billerica, MA, USA). The membranes were blocked in PBST (PBS with $0.1 \%$ Tween-20) containing 5\% non-fat milk for $2 \mathrm{~h}$ at room temperature, and then were incubated with the primary antibodies: Anti-Ki67, anti-proliferation cell nuclear antigen (PCNA), anti-caspase-3, anti-caspase-9, anti-matrix metalloproteinase (MMP)-9, anti-vascular endothelial cell growth factor (VEGF), anti-SOX-4, anti-GAPDH and corresponding HRP-conjugated secondary antibodies. Membranes were extensively washed several times with PBST. Proteins were detected using a ChemiDoc XRS imaging system and Quantity One analysis software (Bio-Rad, San Francisco, CA, USA). GAPDH (Abcam, Cambridge, UK) was used as an endogenous reference. 
Table I. Clinicopathological variables in cervical cancer patients.

\begin{tabular}{lr}
\hline Variable & Cases \\
\hline Age, years ${ }^{\mathrm{a}}$ & \\
$<55$ & 15 \\
$\geq 55$ & 10 \\
Sex & \\
Female & 25 \\
Pathological classification & \\
Squamous classification & 16 \\
Adenoma & 7 \\
Other & 2 \\
Clinical stage & \\
0 & 3 \\
I & 7 \\
II & 10 \\
III & 4 \\
IV & 1 \\
Response to chemotherapy & 1 \\
No or poor & \\
Moderate & 12 \\
Chemotherapy regimen & 5 \\
5-fluorouracil & \\
Cyclophosphamide & \\
Cisplatin & \\
Doxorubicin & \\
\hline
\end{tabular}

${ }^{\mathrm{a} A g e}$ mean \pm standard deviation, 53.2 \pm 11.4 ; range, 48-71.

Luciferase reporter assays. The 3'-UTR of BCYRN1 was PCR amplified from human genomic DNA, and cloned downstream of the firefly luciferase coding region of the pMIR-GLO ${ }^{\mathrm{TM}}$ Luciferase vector (Promega,Madison, WI, USA). The recombinant vector was called pMIR-BCYRN1-wild-type. Mutations in miR-138 binding sites were introduced by site-directed mutagenesis and the resulting vector was called pMIR-BCYRN-mutant. Cells were seeded into 24-well plates and co-transfected with $200 \mathrm{ng}$ of pMIR-BCYRN1 or pMIR-BCYRN1-mut vector and miR-138 mimic or miR-138 inhibitor. Cells were harvested and then lysed (lysis buffer; Promega), after $36 \mathrm{~h}$. The luciferase reporter gene assay was detected by the Dual-Luciferase Reporter Assay system (Promega, Shanghai, China), according to the manufacturer's instructions.

Invasion and migration assays. For the invasion assay, after $48 \mathrm{~h}$ of transfection, $2 \times 10^{4} \mathrm{HeLa}$ cells were cultured without FBS in the upper chamber of an insert (Chemicon, Temecula, CA, USA). Matrigel (Sigma-Aldrich; Merck KGaA, Darmstadt, Germany) was used to precoat the chamber. Then the lower chambers were incubated for $24 \mathrm{~h}$ in culture medium with $10 \%$ FBS, before examination. On the upper surface, HeLa cells were scraped and washed away, whereas the invaded cells on the lower surface were fixed and stained with Diff-Quik (Sysmex, Kobe, Japan) at RT for 2 h. The invading cells were observed and counted under a DMLB2 light microscope (Leica, Wetzlar, Germany) at a magnification of x200 in 10 random fields in each well. For the migration assay, approximately $1.5 \times 10^{6}$ cells/well pretreated with BCYRN1 siRNA or siRNA-scramble were seeded in 6-well plate and cultured overnight until the cells reached $90 \%$ confluence. Then a straight scratch was created by a sterile pipette tip. After rinsing off the destroyed cells with PBS, the plate was cultured in medium for another $24 \mathrm{~h}$. Cell migration was observed using an Olympus IX 70 microscope and imaged at 0 and $24 \mathrm{~h}$ with a digital camera (Leica DFC300FX).

Subcutaneously xenografted mouse model. All animal experiments were carried out in accordance with a protocol approved by the Institutional Animal Care and Use Committee (IACUC). HeLa cells were transfected with BCYRN1 siRNA or siRNA-scramble for $24 \mathrm{~h}$. Then, $4 \times 10^{6}$ cells were subcutaneously inoculated into 6-8 weeks old male athymic nude mice. After tumors (100-150 $\mathrm{mm}^{3}$ ) had established, the tumor volume was measured every 5 days using the same protocol, and calculated in length $\mathrm{x}\left(\right.$ width $\left.^{2}\right) / 2$.

Immunohistochemistry. Formalin-fixed paraffin-embedded sections $(5 \mu \mathrm{M})$ from tissue microarrays were prepared. They were deparaffinized in xylene and rehydrated then were incubated in $30 \% \mathrm{H}_{2} \mathrm{O}_{2}$ to quench the activity of endogenous peroxidase. Then the sections were incubated with primary antibodies directed against MMP-2 and VEGF overnight at $4^{\circ} \mathrm{C}$. Proteins were visualized under a light microscopy.

Statistical analysis. All data were processed using SPSS 13.0 (SPSS, Inc., Chicago, IL, USA). For statistical analysis, quantitative data from at least three experiments were compared and are expressed as the mean \pm SD. Analysis of variance (ANOVA) was used for comparison among groups. $\mathrm{P}<0.05$ was considered to indicate a statistically significant difference.

\section{Results}

BCYRN1 is upregulated in cervical cancer. BCYRN1 relative expression was evaluated using RT-PCR and northern blot from 25 cervical cancer patients. BCYRN1 was upregulated in cervical cancer tissues compared with normal tissues (Fig. $1 \mathrm{~A}$ and $\mathrm{B} ; \mathrm{P}<0.01$ ). Then, we examined the mRNA expression level of BCYRN1 in cervical cancer cells. The non-cancerous ectocervical epithelial cell line (Ect1/E6E7) was used as normal control. Among the cervical cancer cells, the BCYRN1 expression were notably upregulated as compared with that in the Ect1/E6E7 cells (Fig. 1C and D; $\mathrm{P}<0.01)$. We selected HeLa cells for BCYRN1 knockdown and the following experimental study, as it harbored the highest expression level of BCYRN1.

Silencing BCYRN1 attenuates the viability and motility of cervical cancer. Then we evaluated the in vitro effects of BCYRN1 in cervical cancer by transfection of 3 different siRNAs of BCYRN1 into HeLa cells. Then an optimal 


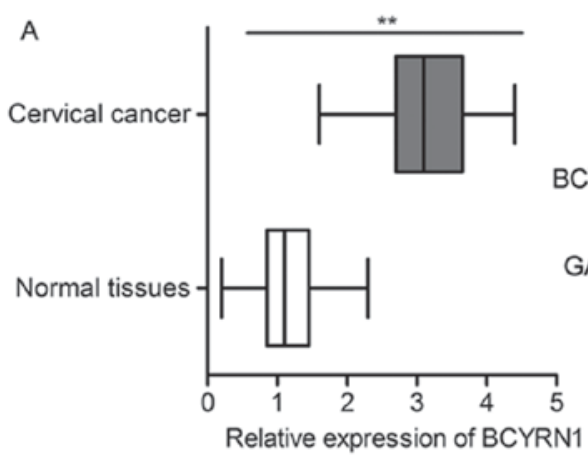

c

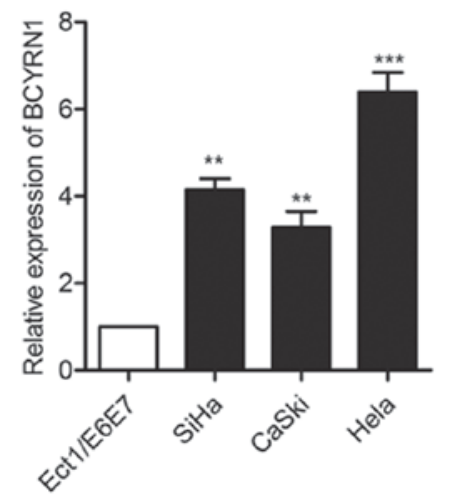

B

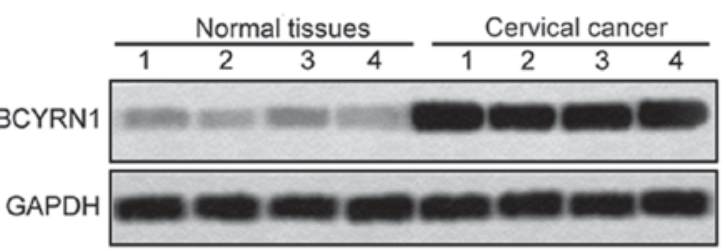

$\mathrm{D}$

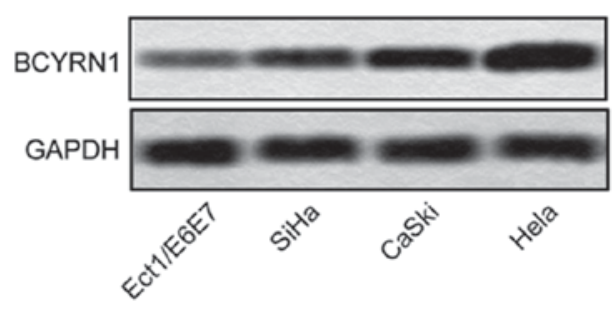

Figure 1. Level of BCYRN1 was upregulated in cervical cancer. (A) Relative expression of BCYRN1 in cervical cancer tissues and adjacent histologically normal tissues was detected by qRT-PCR ( ${ }^{* *} \mathrm{P}<0.01$ vs. normal tissues). (B) Expression of BCYRN1 in cervical cancer tissues and adjacent histologically normal tissues was detected through northern blotting. GAPDH was used as an endogenous reference. (C) Expression of BCYRN1 in three human cervical cancer cell lines ( $\mathrm{SiHa}, \mathrm{HeLa}$ and CaSki) and non-cancerous ectocervical epithelial cell line (Ect1/E6E7) was detected through qRT-PCR $\left({ }^{* *} \mathrm{P}<0.01\right.$ vs. Ect1/E6E7; ${ }^{* * *} \mathrm{P}<0.01$ vs. Ect1/E6E7). (D) Expression of BCYRN1 in related cervical cancer cell lines and esophageal epithelial cell line was valued through northern blotting. GAPDH was used as an endogenous reference. Bars showed means \pm SD of three independent experiments. BCYRN1, brain cytoplasmic RNA 1.

knockdown siRNA (siRNA_1) was chosen in this study. The BCYRN1 siRNA caused a significant downregulation of the mRNA expression of BCYRN1 (Fig. 2A and B; $\mathrm{P}<0.01$ ). Flow cytometric analysis showed that HeLa cells apoptosis was markedly increased after transfection with BCYRN1 siRNA (Fig. $2 \mathrm{C}$ and $\mathrm{D} ; \mathrm{P}<0.01$ ). Next, CCK-8 assay was performed to detect the cell viability. Data showed that transfection with BCYRN1 siRNA distinctly suppressed the proliferation of HeLa cells (Fig. 2E; P<0.05). Given that the inhibition of BCYRN1 reduced cell viability in HeLa cells, further experiments was conducted to examine the effect of BCYRN1 on cell motility. The result of transwell invasion assay showed that invasion cells was noticeably declined in HeLa cells transfected with BCYRN1siRNA $(\mathrm{P}<0.001$; Fig. 3A and B). By comparing the closure of the gap at 0 and $24 \mathrm{~h}$ later after transfection, a significantly decreased closing rate of scratch wounds was detected in BCYRN1 siRNA group compared with the siRNA scramble group $(\mathrm{P}<0.05$; Fig. $3 \mathrm{C}$ and $\mathrm{D})$. The results above indicated that inhibition of BCYRN1 suppressed cell viability and motility in cervical cancer.

MiRNA-138 is downregulated in cervical cancer. As shown in Fig. 4A and B, there was a 3-4-fold decrease in the expression level of miR-138 in the 25 tumors than the normal tissues $(\mathrm{P}<0.01)$. miR-138 level also was downregulated in cervical cancer cell lines compared with noncancerous ectocervical epithelial cell Ect1/E6E7 (Fig. 4C and D; P<0.01). Above data indicated that miR-138 may be as an important role in cervical cancer.

Identification of potential BCYRN1-targeting miRNA-138. According to the prediction results, we identified that BCYRN1 contained five seed sequences of miR-138 (Fig. 5A). That meant miRNA-138 may be a potential targeting of BCYRN1. BCYRN1 was then overexpressed by transfecting lncRNA BCYRN1 into HeLa cells. It showed that overexpression of BCYRN1 could restrain the expression of miR-138 (Fig. 5B). But the level of miR-138 was upregulated after silencing of BCYRN1 (Fig. 5C). Luciferase reporter constructs were generated to further confirm the direct binding between BCYRN1 and miRNA-138. We observed that miRNA-138 mimic decreased the luciferase activities of BCYRN1 wt reporter vector. But luciferase activities in cells transfected with BCYRN1 mut and the miRNA-138 mimic were almost comparable to that of control cells (Fig. 5D; $\mathrm{P}<0.01$ ). Trend relation of BCYRN1 and miR-138 in 25 patients indicated that BCYRN1 and miR-138 showed the reverse changes (Fig. 5E; $\mathrm{P}<0.001)$. It suggests that the regulation between miRNA-138 and BCYRN1 may be in a way similar to the miRNA-mediated silencing of protein-coding genes. These results confirmed the direct binding between BCYRN1 and miRNA-138 and negative regulation relationship.

Bucking effect of miR-138 inhibitor on cervical cancer cell proliferation, invasion and migration. To explore the 

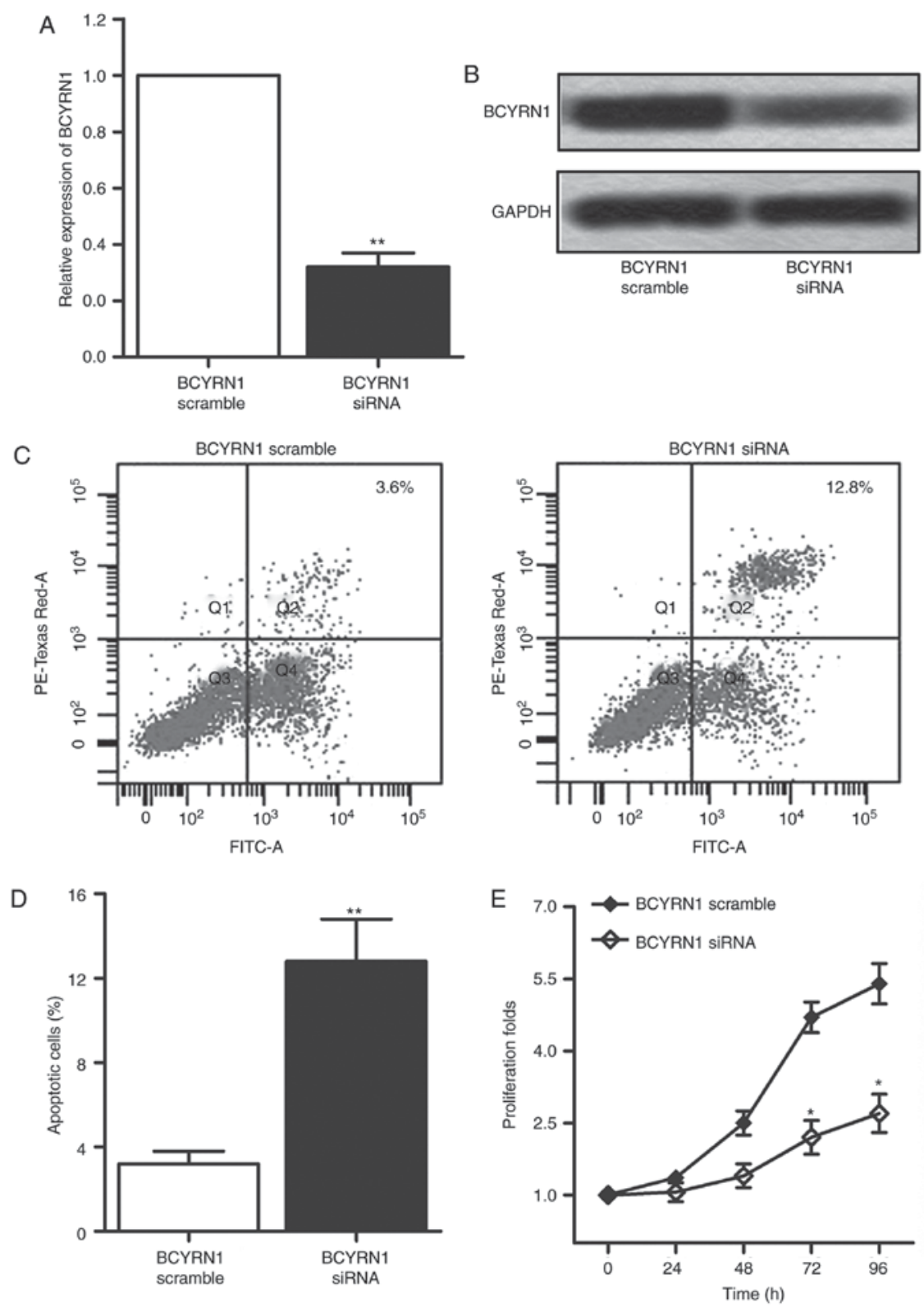

Figure 2. Silencing BCYRN1 inhibits cell proliferation and promotes cell apoptosis of cervical cancer. HeLa cells were transfected with BCYRN1 siRNA or siRNA scramble, respectively. (A) Relative expression of BCYRN1 in HeLa cells was detected through qRT-PCR ("P<0.01 vs. scramble group). (B) Expression of BCYRN1 in HeLa cells was detected through northern blotting. GAPDH was used as an endogenous reference. (C) Cell apoptosis was detected using the flow cytometry. (D) Histogram represents the statistical analysis of cell apoptosis rate. (E) Numbers of cells per well in HeLa cells were measured through CCK-8 assay at $570 \mathrm{~nm}$. The statistical results were shown in the form of a line chart. Bars showed means \pm SD of three independent experiments. ${ }^{*}<0.05$ and ${ }^{* *} \mathrm{P}<0.01$ vs. BCYRN1 scramble group. BCYRN1, brain cytoplasmic RNA 1.

underlying mechanism of such a negative regulation between miR-138 and BCYRN1, we detected the proliferation, apoptosis, invasion and migration in HeLa cells, in response to BCYRN1 knockdown and miR-138 inhibitor. As illustrated in Fig. 6, BCYRN1 siRNA significantly restrained the proliferation, invasion and migration ability of HeLa cells, but promoted apoptosis. The adding of miRNA-138 inhibitor enhanced cell proliferation, invasion and migration, but BCYRN1 siRNA had bucking effect for miRNA-138 inhibitor in HeLa cells $(\mathrm{P}<0.01)$.

Negative regulation of miRNA-138 in the oncogenic activity of BCYRN1 in vivo. To provide more evidence to the idea that BCYRN1's oncogenic activity is in part through the negative regulation of miRNA-138, HeLa cells were transfected with BCYRN1 siRNA. The cells were then injected subcutaneously into nude mice. After cancer cell inoculation, mice were killed 4 weeks. We observed a decrease in tumor growth in the BCYRN1 siRNA group, compared with the BCYRN1 scramble group (Fig. 7A and B; $\mathrm{P}<0.05$ ). Furthermore, the expression level of BCYRN1 from the BCYRN1 siRNA group was lower but miR-138 was significantly increased, compared with BCYRN1 scramble group (Fig. 7C-E; $\mathrm{P}<0.001$ ). As shown in Fig. 7F, expression of MMP-2 and VEGF, which are migration marker proteins, were all reduced in BCYRN1 siRNA group. It indicated that BCYRN1 siRNA depressed cell migration. All these data verified the previous results. 

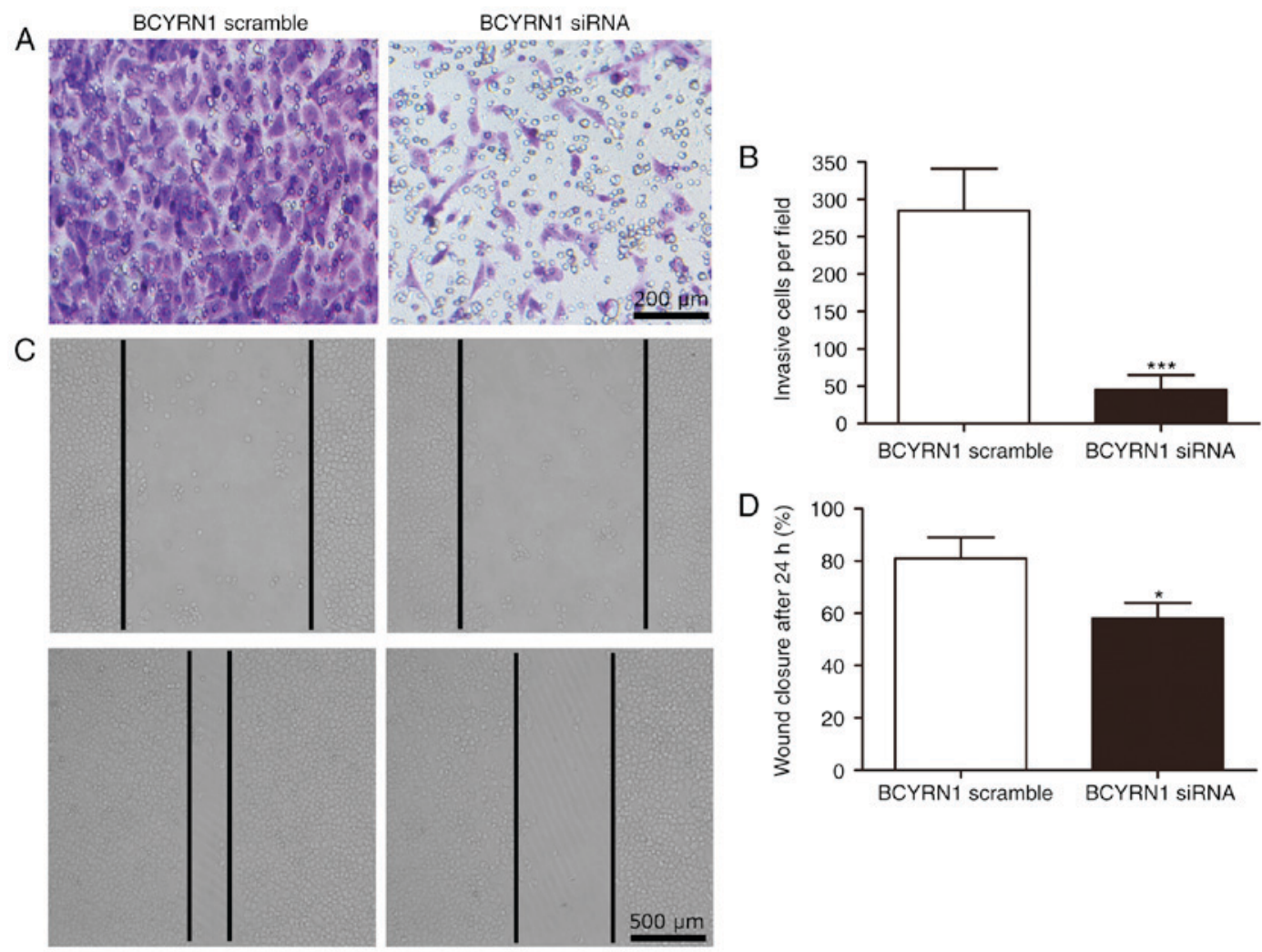

Figure 3. Silencing BCYRN1 inhibits cell invasion and migration of cervical cancer. (A) Transwell invasion assay was conducted to observe the invasive cells in HeLa cells. (B) Histogram represents the statistical analysis of Transwell invasion assay. (C) Migration rate of HeLa cells was observed through wound-healing assays. (D) Histogram represents the statistical analysis of wound-healing assays. ${ }^{*} \mathrm{P}<0.05$ and ${ }^{* * *} \mathrm{P}<0.001$ vs. BCYRN1 scramble group. BCYRN1, brain cytoplasmic RNA 1.

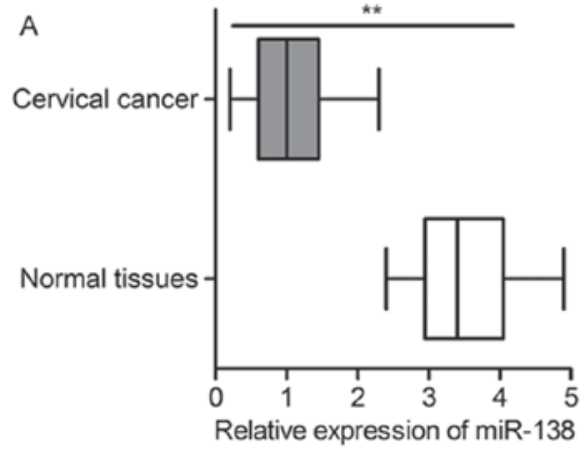

C

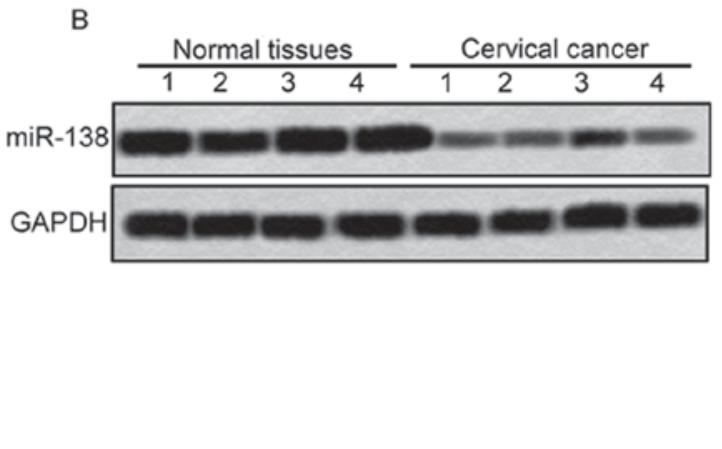

D
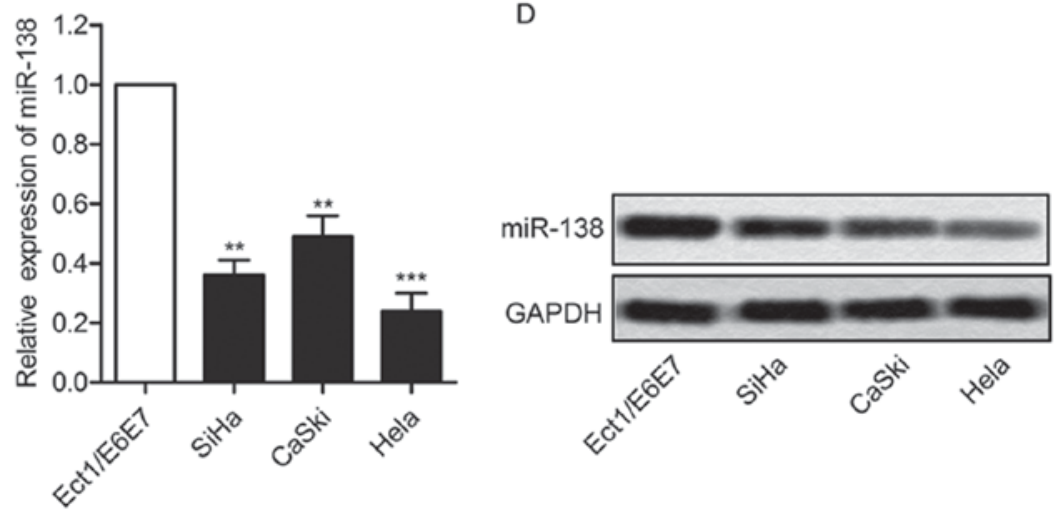

Figure 4. Level of miRNA-138 was downregulated in cervical cancer. Relative expression of miRNA-138 in cervical cancer tissues and adjacent histologically normal tissues was detected by qRT-PCR (A) and northern blotting (B) ( ${ }^{* *} \mathrm{P}<0.01$ vs. normal tissues). (C) Relative expression of miRNA-138 in three human cervical cancer cell lines (SiHa, HeLa and CaSki) and non-cancerous ectocervical epithelial cell line (Ect1/E6E7) was detected through qRT-PCR ( ${ }^{* *}$ P $<0.01$ vs. Ect1/E6E7; ${ }^{* * *} \mathrm{P}<0.01$ vs. Ect1/E6E7). (D) Expression of miR-138 in related tissues and cell lines was measured through northern blotting. GAPDH was used as an endogenous reference. Bars showed means \pm SD of three independent experiments. 
A

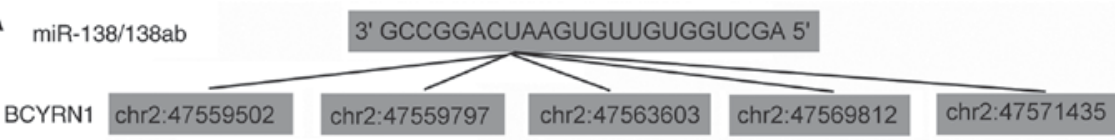

miR-138/138ab 3' GCCGGACUAAGUGUUGUGGUCGA 5'

BCYRN1 3 '........cccucaaagcaACAACccccccc.......5'
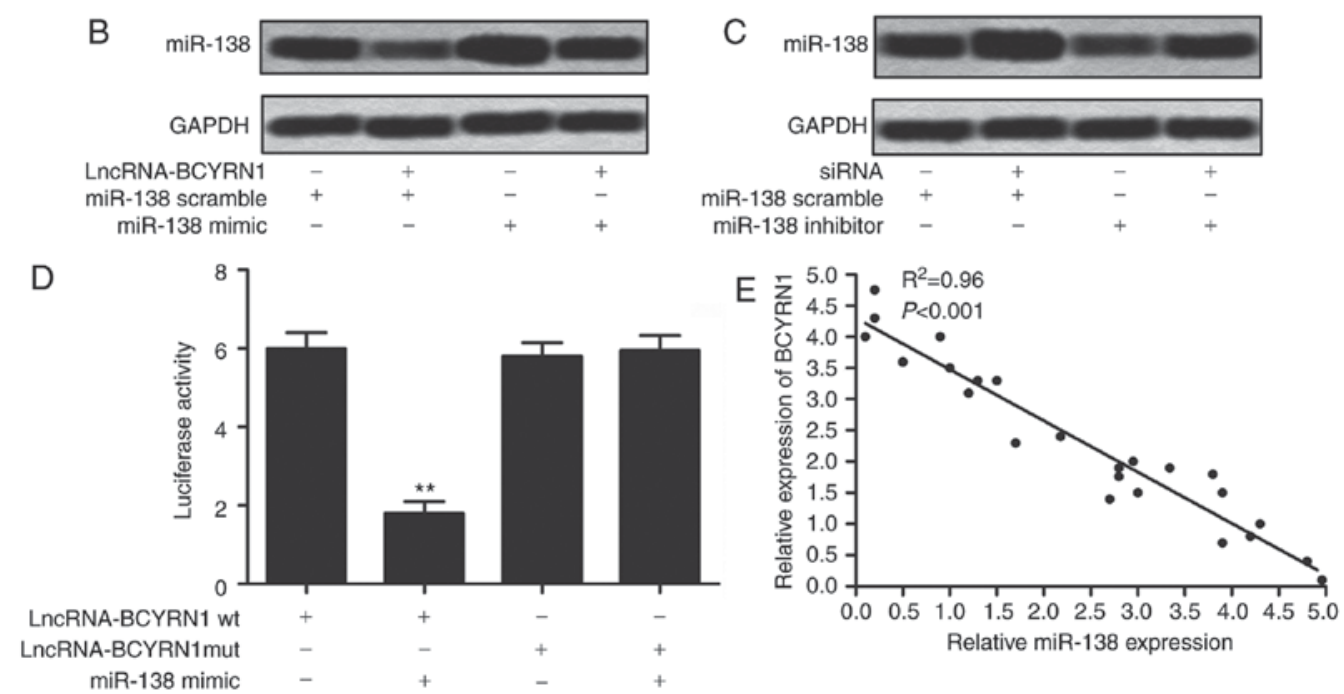

Figure 5. Identification of miRNA-138 as a target of BCYRN1. (A) Alignment of potential BCYRN1 base pairing with miRNA-138 as identified by starBase v2.0. (C) HeLa cells were transfected with lncRNA BCYRN1 and/or miR-138 mimic or mimic control. Expression of miR-138 was detected through northern blotting. (D) Wild-type (lncR-BCYRN1-WT) or mutant (lncR-BCYRN1-Mut) luciferase reporter and/or miR-138 mimic were co-transfected into HeLa cells. Luciferase reporter assay was conducted to detect the luciferase activity in HeLa cells. Bars showed means \pm SD of three independent experiments. (E) Relative expression of BCYRN1 and miR-138 in 25 paired cases of cervical cancer tissues were detected through qRT-PCR. ${ }^{* *} \mathrm{P}<0.01$ vs. IncRNA-BCYRN1 wt group. BCYRN1, brain cytoplasmic RNA 1.
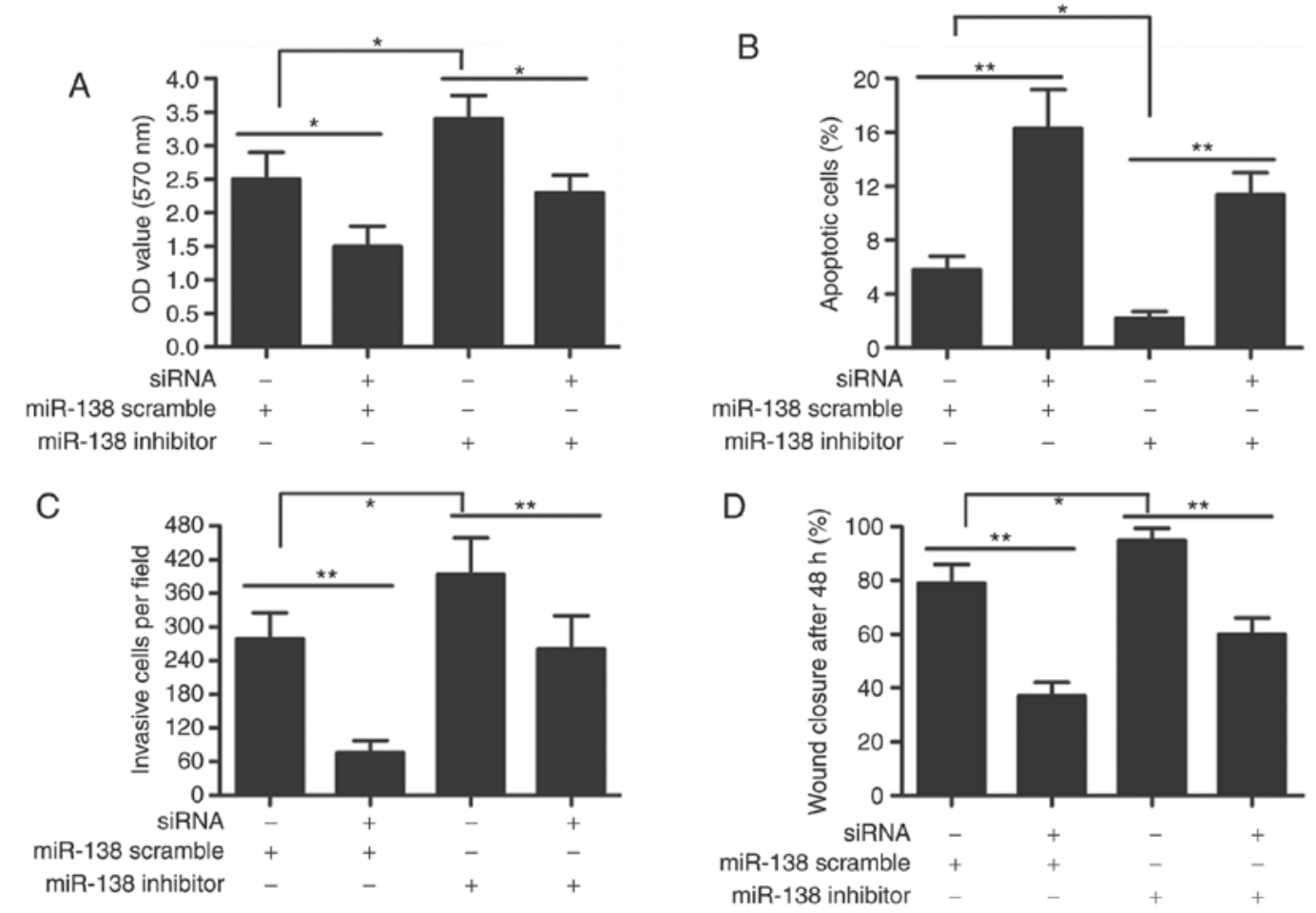

Figure 6. miR-138 inhibitor has bucking effect on BCYRN1 for cell proliferation, invasion and migration. HeLa cells were transfected with BCYRN1 siRNA and/or miR-138 inhibitor or inhibitor control, respectively. (A) OD value in HeLa cells was measured through CCK-8 assay at $570 \mathrm{~nm}$. (B) Cell apoptosis rate was detected through flow cytometry. (C) Cell motility was examined through transwell invasion assay. (D) Cell motility was examined through wound healing assay. ${ }^{*} \mathrm{P}<0.05$ and ${ }^{* *} \mathrm{P}<0.01$ vs. corresponding control group. BCYRN1, brain cytoplasmic RNA 1. 

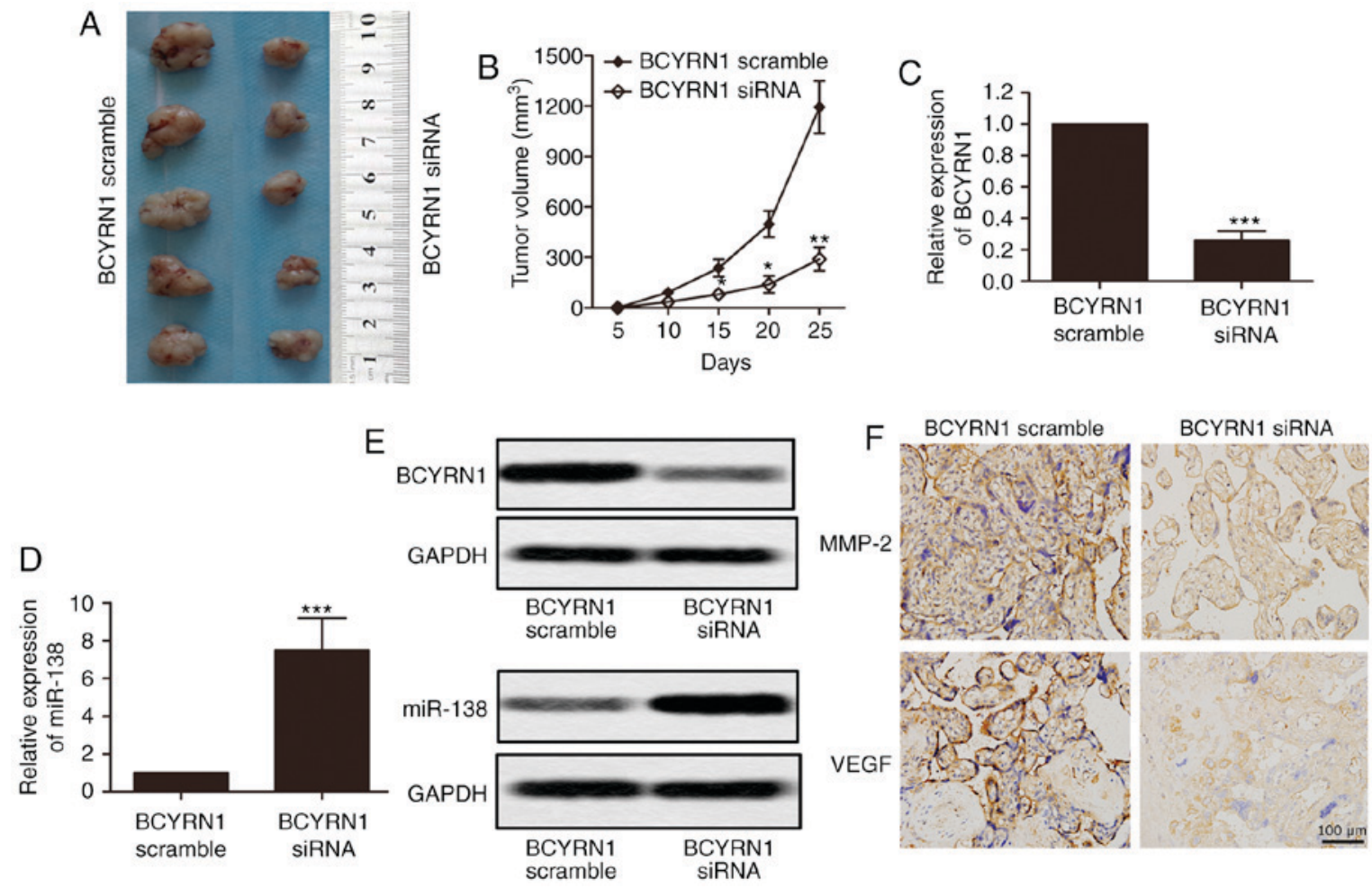

Figure 7. Negative regulation of miRNA-138 in the oncogenic activity of BCYRN1 in vivo. HeLa cells were pre-treated with BCYRN1 siRNA and/or miR-138 inhibitor or scramble, respectively. The cervical cancer xenograft mouse model was created by subcutaneous injection of recombinant cell lines to SPF nude mice. (A) Representative images of cervical cancer xenograft tissues were presented here ( $\mathrm{n}=5$ ). (B) Diameter of cervical cancer xenografts tissues in every group was measured every 5 days from tumor formation to 30 days. Tumor growth curve was shown as means $\pm \mathrm{SD}\left({ }^{*} \mathrm{P}<0.05, * \mathrm{P}<0.05\right.$, ${ }^{* *} \mathrm{P}<0.01$, vs. $\mathrm{scramble}$ group) (C-E) Expression of BCYRN1 and miR-138 in cervical cancer xenograft tissues was detected through qRT-PCR and northern blotting. (F) Migration marker proteins MMP-2 and VEGF were detected by immunohistochemical analysis. GAPDH was used as an endogenous reference. Bars showed means \pm SD of three independent experiments. ${ }^{* * *} \mathrm{P}<0.001$ vs. BCYRN1 scramble group. BCYRN1, brain cytoplasmic RNA 1; MMP, matrix metalloproteinase.

\section{Discussion}

As one of the most common diseases, cervical cancer is seen to be a major cause of women mortality worldwide. Exploring the molecular mechanisms of tumorigenesis is conducive to develop new therapeutics for cervical carcinoma. In this study, it is the first time to explore the expression pattern and biological function of lncRNA BCYRN1 and miR-138 in cervical cancer.

There have been studies showing that lncRNAs are very important in cancer pathogenesis and can provide new insights into the therapy $(16,17)$. Over the past years, the researches about miRNAs have dominated the field of IncRNA regulation $(17,18)$. Nevertheless, the function of lncRNAs in the tumorigenesis of cervical cancer is still unknown. Understanding the precise molecular mechanism would accelerate the development of lncRNA-directed diagnostics and therapeutics against cancers. LncRNA BCYRN1 is normally not detected in normal tissue. However, in this study we detected a low BCYRN expression level in normal cervix tissues. Overexpression of BCYRN1 both in cervical cancer tissues and cell lines were confirmed using RT-PCR and northern blotting. The abnormal expression of cancer metastasis-associated lncRNA may drive tumorigenesis via regulating suppressive and oncogenic pathways. In order to make a further exploration, the BCYRN1 siRNA was transfected into HeLa cells. Data showed that BCYRN1 siRNA depressed cell proliferation, invasion and migration, but apoptotic cells were increased. However, there might be a limitation of the present study that unpredictable off-target effects often happen in siRNAs transfection experiments. Therefore, more siRNA oligonucleotides for BCYRN1 knockdown will be required to verify the effects of BCYRN1 knockdown on HeLa cells in further research.

miRNAs are considered as a group of small non-coding RNA molecules that exhibit different roles in some biological behaviors, for instance, cell apoptosis, differentiation, and tumorigenesis (19). Recently some studies have reported the role of miRNAs in modulating invasion and metastasis of cervical cancer cells. For instance, Deng et al (20) discovered that miRNA-142-3p restrained cervical cancer cells proliferation and invasion, indicating a potential therapeutic approach for cervical cancer (20). Another study revealed that miR-3156-3p is reduced in cervical cancer, suggesting as a tumor-suppressive miRNA (21). miR-138 has been reported to be inhibited in some cancers, including aggressive papillary thyroid carcinoma, head and neck squamous cell carcinoma, and lung cancer tumors $(22,23)$. Literature research reported that miR-138 inhibits proliferation, migration and invasion of cervical cancer cells by targeting hTERT $(24,25)$. In this study, we get similar results that miRNA-138 was evidently inhibited in the cervical cancer tissues and cells. Taken together, combining these literature reports and data we inferred that miR-138 performed as a tumor suppressor in cervical cancer. However, the biological role of miR-138 in cervical cancer had not been explored. Thus the next study aimed to investigate the biological function of miR-138. 
A growing number of reports suggest there is a widespread interaction network between lncRNAs and miRNAs. lncRNAs could modulate RNA by binding and titrating off their binding sites on protein coding messengers (26). An example of this type is lncRNA HULC, highly upregulated in liver cancer. Its upregulation has inhibitory effects on the expression and activity of miRNA-372 (27). Ma et al (28) revealed that IncRNA CCAT1 enhances gallbladder cancer development via negative modulation of miRNA-218-5p. Liz et al focus on the interplay between lncRNAs and miRNAs, and find the importance of such interactions in the tumorigenic process, providing new insight into the regulatory mechanisms of several ncRNA classes in cancer (26). Similar report suggests that miRNA-29 can regulate expression of the lncRNA gene MEG3 in hepatocellular cancer and may contribute to human hepatocellular cancer growth (29). Similar phenomenon was observed between MALAT1 and miR-145 (30). The interaction between IncRNA and miRNA remains to be explored.

We made a study for miRNAs that had complementary base pairing with lncRNA BCYRN1 and fortunately some miRNAs were identified. We focused on miRNA-138, as it has biological function in cervical cancer. Inhibiting BCYRN1 increased miRNA-138 expression, while ectopic expression of BCYRN1 induced the downregulation of miRNA-138 and the miRNA-138-binding site is requisite for the BCYRN1-mediated repression. As we mentioned earlier recent reports indicate lncRNAs may exert modulatory effect via miRNAs. The miRNA-138 was demonstrated to inhibit tumor growth, while BCYRN1 promoted tumor progression. We explored the biological aspect of BCYRN1 and miRNA-138 in HeLa cells. Our study exhibited that although silencing BCYRN1 expression suppressed the proliferation and migration of HeLa cells, miRNA-138 inhibitor had a reversed-effect for BCYRN1 siRNA. Thus it suggests that BCYRN1 may promote tumor development through regulating miRNA-138. Consequently, we further explored the underlying mechanism between them. The results showed that miRNA-138 inhibitor enhanced cell proliferation, invasion and migration, but BCYRN1 siRNA had bucking effect for miRNA-138 inhibitor on cervical cancer cell.

In conclusion, it demonstrated that BCYRN1 increases cervical cancer cell proliferation and invasion by targeting miR-138 in vitro and in vivo. Our findings show that the regulation between BCYRN1 and miR-138 may represent a valuable therapeutic target for cervical cancer therapy. It suggests another layer of regulation involving lncRNAs and miRNAs in both molecular and biological aspects. Further research is needed to identify the more detailed signal pathways involved in the pathogenesis and metastasis of cervical cancer that are supposedly regulated by BCYRN1 and miR-138.

\section{References}

1. Barra F, Lorusso D, Leone Roberti Maggiore U, Ditto A, Bogani G, Raspagliesi F and Ferrero S: Investigational drugs for the treatment of cervical cancer. Expert Opin Investig Drugs 26: 389-402, 2017.

2. PDQ Screening and Prevention Editorial Board: Cervical cancer prevention $(\mathrm{PDQ} \otimes)$ : Health professional version. In: PDQ Cancer Information Summaries. National Cancer Institute, Bethesda, MD, 2002.
3. Chen J, Shishkin AA, Zhu X, Kadri S, Maza I, Guttman M, Hanna JH, Regev A and Garber M: Evolutionary analysis across mammals reveals distinct classes of long non-coding RNAs. Genome Biol 17: 19, 2016.

4. Seles M,HuttererGC, Kiesslich T,PummerK, Berindan-Neagoe I, Perakis S, Schwarzenbacher D, Stotz M, Gerger A and Pichler M: Current insights into long non-coding RNAs in renal cell carcinoma. Int J Mol Sci 17: 573, 2016.

5. Zheng C, Hao H, Chen L and Shao J: Long noncoding RNAs as novel serum biomarkers for the diagnosis of hepatocellular carcinoma: A systematic review and meta-analysis. Clin Transl Oncol 19: 961-968, 2017.

6. Gomes CC, de Sousa SF, Calin GA and Gomez RS: The emerging role of long noncoding RNAs in oral cancer. Oral Surg Oral Med Oral Pathol Oral Radiol 123: 235-241, 2017.

7. Booy EP, McRae EK, Howard R, Deo SR, Ariyo EO, Dzananovic E, Meier M, Stetefeld J and McKenna SA: RNA helicase associated with AU-rich element (RHAU/DHX36) interacts with the 3'-tail of the long non-coding RNA BC200 (BCYRN1). J Biol Chem 291: 5355-5372, 2016.

8. Hu T and Lu YR: BCYRN1, a c-MYC-activated long non-coding RNA, regulates cell metastasis of non-small-cell lung cancer. Cancer Cell Int 15: 36, 2015.

9. Zhang XY, Zhang LX, Tian CJ, Tang XY, Zhao LM, Guo YL, Cheng DJ, Chen XL, Ma LJ and Chen ZC: LncRNAs BCYRN1 promoted the proliferation and migration of rat airway smooth muscle cells in asthma via upregulating the expression of transient receptor potential 1. Am J Transl Res 8: 3409-3418, 2016.

10. Lin Y, Liu J, Huang Y, Liu D, Zhang G and Kan H: MicroRNA-489 plays an anti-metastatic role in human hepatocellular carcinoma by targeting matrix metalloproteinase-7. Transl Oncol 10: 211-220, 2017.

11. Jafri MA, Al-Qahtani MH and Shay JW: Role of miRNAs in human cancer metastasis: Implications for therapeutic intervention. Semin Cancer Biol 44: 117-131, 2017.

12. Soifer HS, Rossi JJ and Saetrom P: MicroRNAs in disease and potential therapeutic applications. Mol Ther 15: 2070-2079, 2007.

13. Vrana D, Matzenauer M, Aujesky R, Vrba R, Neoral C, Melichar B and Souček P: Potential predictive role of microRNAs in the neoadjuvant treatment of esophageal cancer. Anticancer Res 37: 403-412, 2017.

14. Jiang L, Liu X, Kolokythas A, Yu J, Wang A, Heidbreder CE, Shi F and Zhou X: Downregulation of the Rho GTPase signaling pathway is involved in the microRNA-138-mediated inhibition of cell migration and invasion in tongue squamous cell carcinoma. Int J Cancer 127: 505-512, 2010.

15. O'Donohue MF, Choesmel V, Faubladier M, Fichant G and Gleizes PE: Functional dichotomy of ribosomal proteins during the synthesis of mammalian $40 \mathrm{~S}$ ribosomal subunits. J Cell Biol 190: 853-866, 2010.

16. Qi P and Du X: The long non-coding RNAs, a new cancer diagnostic and therapeutic gold mine. Mod Pathol 26: 155-165, 2013.

17. Prensner JR and Chinnaiyan AM: The emergence of lncRNAs in cancer biology. Cancer Discov 1: 391-407, 2011.

18. Lin XC, Zhu Y, Chen WB, Lin LW, Chen DH, Huang JR, Pan K, Lin Y, Wu BT, Dai Y and Tu ZG: Integrated analysis of long non-coding RNAs and mRNA expression profiles reveals the potential role of lncRNAs in gastric cancer pathogenesis. Int J Oncol 45: 619-628, 2014.

19. Zhao M, Ang L, Huang J and Wang J: MicroRNAs regulate the epithelial-mesenchymal transition and influence breast cancer invasion and metastasis. Tumour Biol 39: 1010428317691682 , 2017. doi: $10.1177 / 1010428317691682$.

20. Deng B, Zhang Y, Zhang S, Wen F, Miao Y and Guo K: MicroRNA-142-3p inhibits cell proliferation and invasion of cervical cancer cells by targeting FZD7. Tumour Biol 36: 8065-8073, 2015

21. Xia YF, Pei GH, Wang N, Che YC, Yu FS, Yin FF, Liu HX, Luo B and Wang YK: miR-3156-3p is downregulated in HPV-positive cervical cancer and performs as a tumor-suppressive miRNA. Virol J 14: 20, 2017.

22. Wang Q, Zhong M, Liu W, Li J, Huang J and Zheng L: Alterations of microRNAs in cisplatin-resistant human non-small cell lung cancer cells (A549/DDP). Exp Lung Res 37: 427-434, 2011.

23. Zhao X, Yang L, Hu J and Ruan J: miR-138 might reverse multidrug resistance of leukemia cells. Leuk Res 34: 1078-1082, 2010.

24. Zhou N, Fei D, Zong S, Zhang M and Yue Y: MicroRNA-138 inhibits proliferation, migration and invasion through targeting hTERT in cervical cancer. Oncol Lett 12: 3633-3639, 2016. 
25. Li B, Yang XX, Wang D and Ji HK: MicroRNA-138 inhibits proliferation of cervical cancer cells by targeting c-Met. Eur Rev Med Pharmacol Sci 20: 1109-1114, 2016.

26. Liz J and Esteller M: lncRNAs and microRNAs with a role in cancer development. Biochim Biophys Acta 1859: 169-176, 2016.

27. Wang J, Liu X, Wu H, Ni P, Gu Z, Qiao Y, Chen N, Sun F and Fan Q: CREB up-regulates long non-coding RNA, HULC expression through interaction with microRNA-372 in liver cancer. Nucleic Acids Res 38: 5366-5383, 2010.

28. Ma MZ, Chu BF, Zhang Y, Weng MZ, Qin YY, Gong W and Quan ZW: Long non-coding RNA CCAT1 promotes gallbladder cancer development via negative modulation of miRNA-218-5p. Cell Death Dis 6: e1583, 2015.
29. Braconi C, Kogure T, Valeri N, Huang N, Nuovo G, Costinean S, Negrini M, Miotto E, Croce CM and Patel T: MicroRNA-29 can regulate expression of the long non-coding RNA gene MEG3 in hepatocellular cancer. Oncogene 30: 4750-4756, 2011.

30. Lu H, He Y, Lin L, Qi Z, Ma L, Li L and Su Y: Long non-coding RNA MALAT1 modulates radiosensitivity of $\mathrm{HR}-\mathrm{HPV}^{+}$cervical cancer via sponging miR-145. Tumour Biology 37: 1683-1691, 2016. 